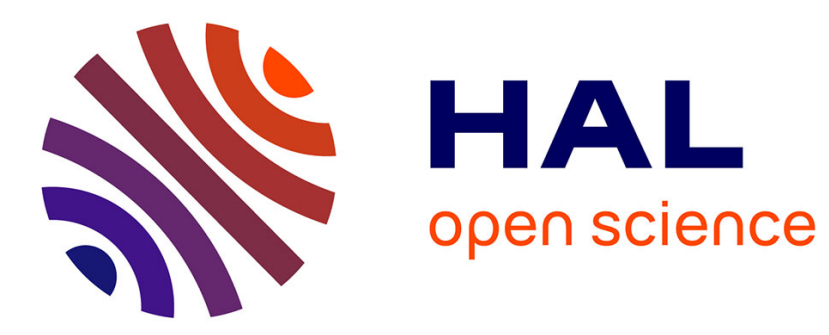

\title{
Thermal diffusivity identification based on an iterative regularization method
}

Lamia Attar, Laetitia Perez, Rémy Nouailletas, Emmanuel Moulay, Laurent

Autrique

\section{- To cite this version:}

Lamia Attar, Laetitia Perez, Rémy Nouailletas, Emmanuel Moulay, Laurent Autrique. Thermal diffusivity identification based on an iterative regularization method. 54th IEEE Conference on Decision and Control, Dec 2015, Osaka, Japan. pp.6, 10.1109/CDC.2015.7402322 . hal-01258555v3

\section{HAL Id: hal-01258555 \\ https://hal.science/hal-01258555v3}

Submitted on 19 Apr 2016

HAL is a multi-disciplinary open access archive for the deposit and dissemination of scientific research documents, whether they are published or not. The documents may come from teaching and research institutions in France or abroad, or from public or private research centers.
L'archive ouverte pluridisciplinaire HAL, est destinée au dépôt et à la diffusion de documents scientifiques de niveau recherche, publiés ou non, émanant des établissements d'enseignement et de recherche français ou étrangers, des laboratoires publics ou privés. 


\title{
Thermal diffusivity identification based on an iterative regularization method*
}

\author{
L. Attar, L. Perez, R. Nouailletas, E. Moulay, and L. Autrique
}

\begin{abstract}
This article deals with the identification of a space and time dependent material thermal diffusivity. Such parameter is involved in heat transfers described by partial differential equations. An iterative regularization method based on a conjugate gradient algorithm is implemented. Such approach is attractive in order to efficiently deal with measurement noises and model errors. Numerical results are illustrated according to several simulations.
\end{abstract}

\section{INTRODUCTION}

Partial differential equations (PDE) systems are commonly used to model thermal phenomena and resulting mathematical models are validated since pioneer works presented in [1]. If one or several model parameters are not known with the required accuracy, it is obvious that predicted results have to be considered suspiciously. In such a context, in the specific situation of a control strategy which has to be synthetized considering predicted system state, a preliminary stage of model identification is crucial. In the proposed study, the identification of a space $x$ and time $t$ dependent thermal diffusivity $\alpha(x, t)$ is investigated.

Since inverse heat conduction problems are ill-posed [2], an iterative regularization method has to be numerically implemented. The proposed method is different from Kalman approach which strongly depends on a priori information such as the noise distribution or the parameterization of the unknown input [3], and from LPV (Linear Parameter-Varying) approach [4]. The method proposed in our communication is based on a Conjugate Gradient algorithm which is an iterative regularization method which is relevant for minimizing the effect of random perturbations in measurement as well as for dealing with model errors [5].

Usually, material thermal diffusivity $\alpha(\theta)$ depends only on temperature $\theta$ [6]. However it may depends on space

\footnotetext{
* This work was partially supported by the ANR-12-BS03-008-03.

L. Attar is with the LARIS, 62 avenue notre dame du lac, 49000 Angers, France (e-mail: lamia.attar@etud.univ-angers.fr)

L. Perez is with the LTN, La Chantrerie, Rue Christian Pauc, BP50609, 44306 Nantes cedex 03, France (email: laetitia.perez@univ-nantes.fr)

R. Nouailletas is with IRFM, CEA Cadarache, 13108 Saint-Paul Lez Durance Cedex, France (email: remy.nouailletas@cea.fr)

E. Moulay is with XLIM-SIC, 10, boulevard Curie, 86962 Futuroscope Chasseneuil Cedex, France (email: emmanuel.moulay@univ-poitiers.fr)

L. Autrique is with the LARIS, 62 avenue notre dame du lac, 49000 Angers, France (corresponding author : phone: +33 244687 518; fax :+33 244687 561; e-mail: laurent.autrique@ univ-angers.fr)
}

$\alpha(x)$, as for functionally graded materials [7]. For specific applications it may depends on space and time, as for instance phase change materials [8] or nuclear fusion plasmas $[9,10]$. In the following, the general situation of a space and time dependent thermal diffusivity $\alpha(x, t)$ is investigated using the Conjugate gradient method (CGM). To the best of our knowledge, this strategy has never been applied to this special case.

The article is organized as follows. In Section II, the studied model is presented and the inverse problem is formulated as a minimization problem. In Section III, the iterative regularization method is detailed and implemented. In the last Section IV, numerical results are provided and effects of a specific model error and measurement noises are investigated.

\section{INVERSE ILL-POSED PROBLEM}

\section{A. Statement}

In thermal context, several kinds of inverse problems related to heat conduction can be encountered [2]: retrospective problem (for initial state reconstruction for example [11]), boundary inverse problem (heating flux identification for example [12]), geometric inverse problem (an illustration to cavity detection is given in [13]) and coefficient inverse problem. We are mainly interested in this last situation and in the determination of a thermophysical property which is space-time dependent.

Let us consider the following notations in order to introduce the direct problem. Space variable is denoted by $x \in[0, L]$ and time variable is $t \in\left[0, t_{f}\right]$. System state is the temperature denoted by $\theta(x, t)$. Evolution of the system (from initial temperature $\theta_{0}$ ) depends on thermal diffusivity usually defined as the ratio of the thermal conductivity $\left[\mathrm{W} \cdot \mathrm{m}^{-1} \cdot \mathrm{K}^{-1}\right]$ versus the volumetric heat $\left[\mathrm{J} \cdot \mathrm{m}^{-3} \cdot \mathrm{K}^{-1}\right]$.

In the following, thermal diffusivity is denoted by $\alpha(x, t)$ in $\left[\mathrm{m}^{2} \cdot \mathrm{s}^{-1}\right]$. System input is the heat flux divided by the volumetric heat and is denoted by $g(x, t)$. Then, if all the parameters are known, direct problem can be expressed as follows: 
Attar L., Perez L., Nouailletas R., Moulay E., Autrique L., Thermal diffusivity identification based on an iterative regularization method, IEEE Conference on Decision and Control 2015, Osaka Japan, December 2015.

Direct problem.

Considering that $\mathbb{P}=\left\{L, t_{f}, \alpha(x, t), g(x, t), \theta_{0}\right\}$

known, find $\theta(x, t)$ solution of the partial differential equations (PDE) system

$$
\begin{cases}\frac{\partial \theta(x, t)}{\partial t}-\frac{\partial}{\partial x}\left(\alpha(x, t) \frac{\partial \theta(x, t)}{\partial x}\right)=g(x, t) & \\ \forall(x, t) \in[0, L] \times\left[0, t_{f}\right] \\ \theta(x, 0)=\theta_{0} \quad \forall x \in[0, L] \\ \theta(0, t)=\theta_{0} ;\left.\frac{\partial \theta(x, t)}{\partial x}\right|_{x=L}=0 & \forall t \in\left[0, t_{f}\right]\end{cases}
$$

Except for academic situations, direct problem is usually solved according to numerical methods such as finite element method [14-17]. In the following, such method is implemented considering Comsol® solver [18]. Let us consider the realistic input parameter listed in Table 1. Thermal diffusivity $\alpha(x, t)$ and heating source $g(x, t)$ are shown in Fig. 1.

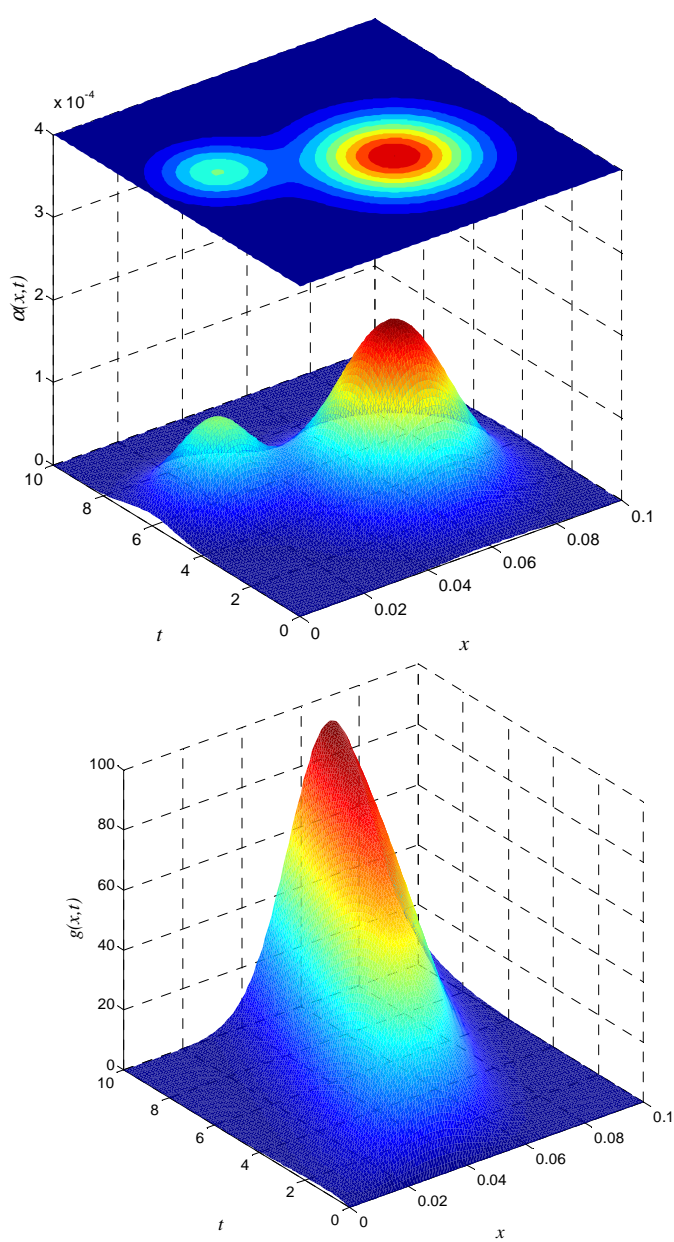

Figure 1. Thermal diffusivity and heating source.
TABLE I. INPUT PRAMETERS FOR DIRECT PROBLEM

\begin{tabular}{ll}
$L=0.1 \mathrm{~m}$ & $\alpha(x, t)=10^{-4} e^{-\frac{\left((x-0.02)^{2}+\frac{(t-6)^{2}}{100}\right)}{2.10^{-4}}}+2.10^{-4} e^{-\frac{\left((x-0.06)^{2}+\frac{(t-4)^{2}}{100}\right)}{4.10^{-4}}}$ \\
\hline$t_{f}=10 \mathrm{~s}$ & $g(x, t)=100\left(1-e^{\left.-\frac{t}{4}\right)} e^{\frac{-\left(x-\left(0.03+0.04 \frac{t}{t_{f}}\right)\right)^{2}}{4.10^{-4}}}\right.$ \\
\hline$\theta_{0}=293 \mathrm{~K}$
\end{tabular}

Considering all the previous input parameters, direct problem is numerically solved. Temperature evolution $\theta(x, t)$ is presented in Fig. 2.
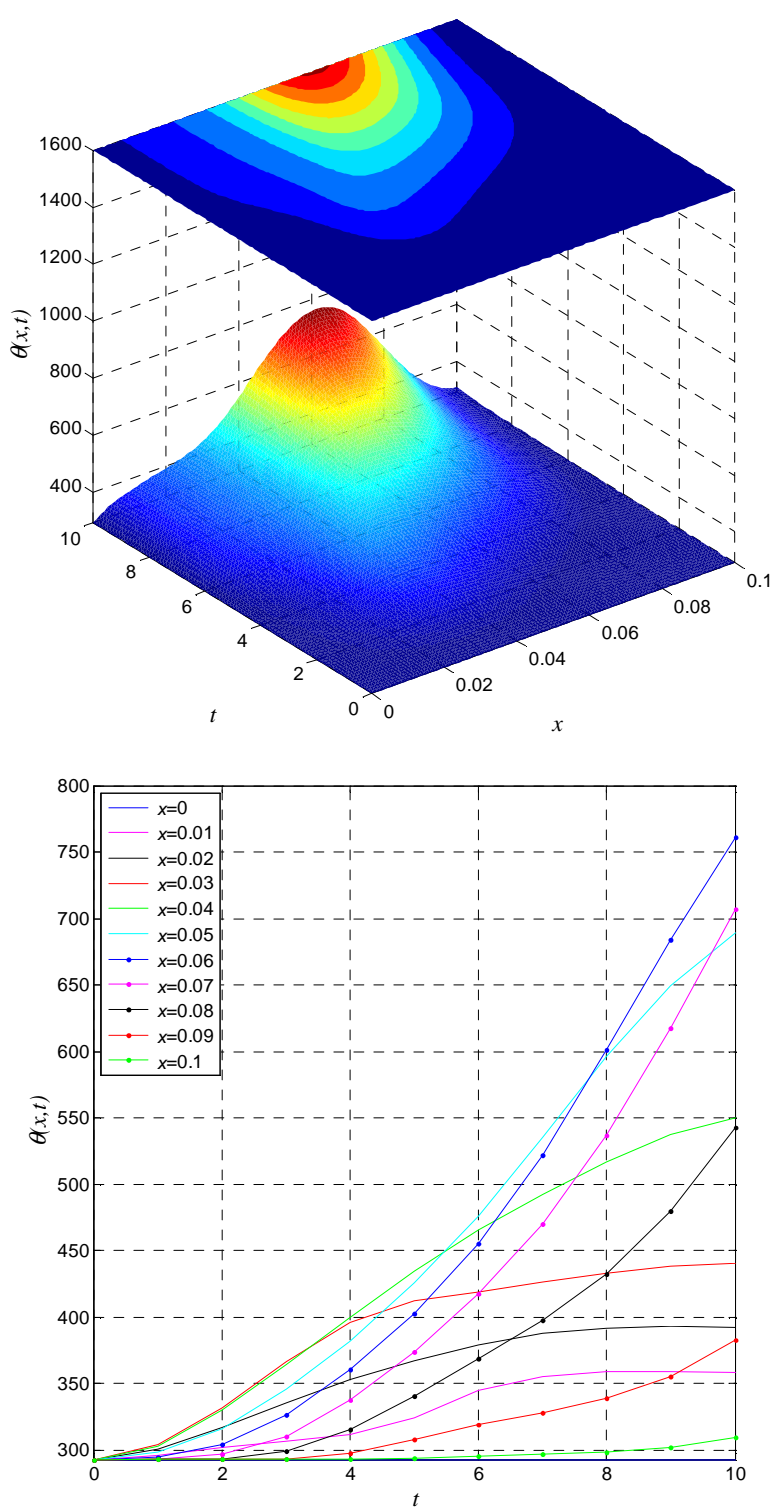

Figure 2. Temperature evolution.

\section{B. Inverse problem formulation}

If one or several input parameter $p \in \mathbb{P}$ is unknown then an inverse problem can be solved considering state 
Attar L., Perez L., Nouailletas R., Moulay E., Autrique L., Thermal diffusivity identification based on an iterative regularization method, IEEE Conference on Decision and Control 2015, Osaka Japan, December 2015.

observations $\hat{\theta}(x, t)$. It is usual to investigate such inversion as a minimization problem where a quadratic cost-function has to be minimized:

Inverse problem.

Find

$$
\begin{aligned}
p^{*} & =\underset{p \in \mathscr{P}}{\arg \min } J(\theta, p) \\
& =\underset{p \in \mathscr{P}}{\arg \min }\left[\iint_{[0, L] \times\left[0, t_{f}\right]}(\theta(x, t ; p)-\hat{\theta}(x, t))^{2} d x d t\right]
\end{aligned}
$$

such that $\theta(x, t ; p)$ is solution of the direct problem obtained with parameter $p$.

Let us consider that the thermal diffusivity $\alpha(x, t)$ has to be identified. In such an aim several temperature measurements are available: $N_{s}$ sensors are located in $[0, L]$ and measured temperatures are denoted by $\hat{\theta}_{i}(t)$ for $i=1, \cdots, N_{s}$. Sensors location are denoted by $x_{i}$. Moreover parametrization of the unknown thermal diffusivity has to be considered. In the absence of a priori information, $\alpha(x, t)$ is assumed without any loss of generalities to be a piecewise linear function in two dimension:

$$
\alpha(x, t)=\sum_{j=1}^{N t} \sum_{i=1}^{N x} \alpha_{i j} s_{i}(x) s_{j}(t)
$$

Where $N t$ and $N x$ are the number of discretization step related to the space and time dependence, $s_{i}(x)$ and $s_{j}(t)$ are basis functions (hat functions). Unknown thermal diffusivity tensor is thus defined considering matrix $\alpha=\left[\alpha_{i j}\right]_{N \times x N_{t}}$. Then, $N=N x . N t$ unknown coefficients have to be identified considering the inverse problem :

Inverse problem.

Find

$$
\begin{aligned}
\alpha^{*} & =\underset{\alpha \in \mathbb{R}^{N \times N N t}}{\arg \min } J(\theta, \alpha) \\
& =\underset{\alpha \in \mathbb{R}^{N \times \times N t}}{\arg \min }\left[\int_{0}^{t_{f}} \sum_{i=1}^{N_{s}}\left(\theta\left(x_{i}, t ; \alpha\right)-\hat{\theta}_{i}(t)\right)^{2} d t\right]
\end{aligned}
$$

such that $\theta(x, t ; \alpha)$ is solution of the direct problem.

Previous inverse heat conduction problem is ill-posed due to the solution instability in the sense of Hadamard [19] since small variations on measurements can induce great variations on $\alpha$. In practice, it is not possible to deal with exact data due to numerical errors induced by finite element resolution and noises in measurements. In such a context, pioneer works have been performed by Tikhonov for solving ill-posed problems. Proposed methods for the construction of stable solutions are named regularization methods [2, 19, 20]. In order to obtain a stable solution, the basic principle is to consider a new problem involving a small parameter so that the new inverse problem is stable. The positive parameter is called the regularization parameter. Construction of regularizers is not trivial and in the next section, convergence of a regularization algorithm is discussed.

\section{ITERATIVE REGULARIZATION METHOD}

In order to obtain a stable solution, well-posed problems have to be solved at each iteration of the minimization algorithm. For usual descent methods, at each iteration a new value of the unknown parameter is obtained

$$
\alpha^{k+1}=\alpha^{k}+\Delta\left(\alpha^{k}\right)
$$

where the correction $\Delta\left(\alpha^{k}\right)$ at each iteration is chosen such that $J\left(\alpha^{k+1}\right)<J\left(\alpha^{k}\right)$.

In [2], O.M Alifanov states that "such a method of damping the instability when specifying an approximate solution for an ill-posed problem is based on viscous properties of numerical algorithms of optimization".

Iterative minimization of quadratic cost function $J$ based on CGM is known as a stable algorithm for inverse heat conduction problem. In [21], stabilizing effect during the iterative minimization is highlighted. In an academic situation in a 1D geometry, analytical solution of direct problem is formulated. Then, it is shown that the main structure of the boundary heat flux is estimated in the first iterations. The CGM acts like a sequential filtering mechanism capable of rejecting random perturbations in measurements during the identification process. Iteration number acts as a regularization parameter. CGM algorithm can be presented as follows [22]:

- Step 1: initialization $(k=0)$ of $\boldsymbol{\alpha}^{k}$.

- Step 2: estimation of the cost function $J\left(\alpha^{k}\right)$,

if $J\left(\alpha^{k}\right) \leq J_{\text {stop }}$ then $\alpha^{k}$ is a correct estimation of the unknown thermal diffusivity and the algorithm is stopped ;

else goto step 3

- Step 3: evaluation of the cost function gradient $\left(\frac{\partial J^{k}}{\partial \alpha_{i j}^{k}}\right)$ for $i=1, \cdots, N_{x}$ and $j=1, \cdots, N_{t}$. Then, estimation of the descent direction $\boldsymbol{D}^{k}$.

- Step 4: evaluation of the descent depth $\gamma^{k} \in \mathbb{R}$ related to the descent direction $\boldsymbol{D}^{k}$

- Step 5: estimation of the new parameter $\boldsymbol{\alpha}^{k+1}=\boldsymbol{\alpha}^{k}+\boldsymbol{\gamma}^{k} \boldsymbol{D}^{k}$ then $(k=k+1)$ goto step 2 .

Most important stages are the gradient calculation and the descent depth estimation. Both numerical resolutions are obtained considering PDE systems well posed in Hadamard sense.

The first system leads to gradient estimation: 
Attar L., Perez L., Nouailletas R., Moulay E., Autrique L., Thermal diffusivity identification based on an iterative regularization method, IEEE Conference on Decision and Control 2015, Osaka Japan, December 2015.

\section{Adjoint problem.}

Find $\Psi^{k}(x, t)$ solution of the PDE system

$$
\begin{cases}\frac{\partial \Psi^{k}}{\partial t}+\frac{\partial}{\partial x}\left(\alpha^{k} \frac{\partial \Psi^{k}}{\partial x}\right)=E^{k} & \forall(x, t) \in[0, L] \times\left[0, t_{f}\right] \\ \Psi^{k}\left(x, t_{f}\right)=0 & \forall x \in[0, L] \\ \psi^{k}(0, t)=0 ;\left.\frac{\partial \Psi^{k}(.)}{\partial x}\right|_{x=L}=0 & \forall t \in\left[0, t_{f}\right]\end{cases}
$$

where $E^{k}(x, t)=\sum_{i=1}^{N_{s}}\left(\theta\left(x_{i}, t ; \alpha\right)-\hat{\theta}_{i}(t)\right) \delta_{D x_{i}}(x)$ and $\delta_{D x_{i}}$ is the Dirac distribution related to sensor $i$.

According to the previous notation, gradient is defined considering the matrix $\nabla \boldsymbol{J}^{k}=\left[\frac{\partial J^{k}}{\partial \alpha_{i j}^{k}}\right]_{N_{x} \times N_{t}}$ with :

$$
\left(\frac{\partial J^{k}}{\partial \alpha_{i j}^{k}}\right)=\iint_{[0, L] \times\left[0, t_{f}\right]} \frac{\partial \theta^{k}(x, t)}{\partial x} \frac{\partial \Psi^{k}(x, t)}{\partial x} s_{i}(x) s_{j}(t) d x d t
$$

Descent direction is defined as:

$$
\boldsymbol{D}^{k}=\left[d_{i j}^{k}\right]_{N_{x} \times N_{t}}=-\nabla \boldsymbol{J}^{k}+\beta^{k} \boldsymbol{D}^{k-1}
$$

where $\beta^{k}=\frac{\left\|\nabla \boldsymbol{J}^{k}\right\|^{2}}{\left\|\nabla \boldsymbol{J}^{k-1}\right\|^{2}}\left(\right.$ except for $\left.\beta^{0}=0\right)$. Norm $\|$.$\| is the$ Frobenius matrix norm $\|\boldsymbol{M}\|=\sqrt{\sum_{j=1}^{N t} \sum_{i=1}^{N x} M_{i j}^{2}}$.

The second PDE system leads to the descent depth estimation:

Sensitivity problem.

Find $\delta \theta^{k}(x, t)$ solution of the partial differential equations (PDE) system:

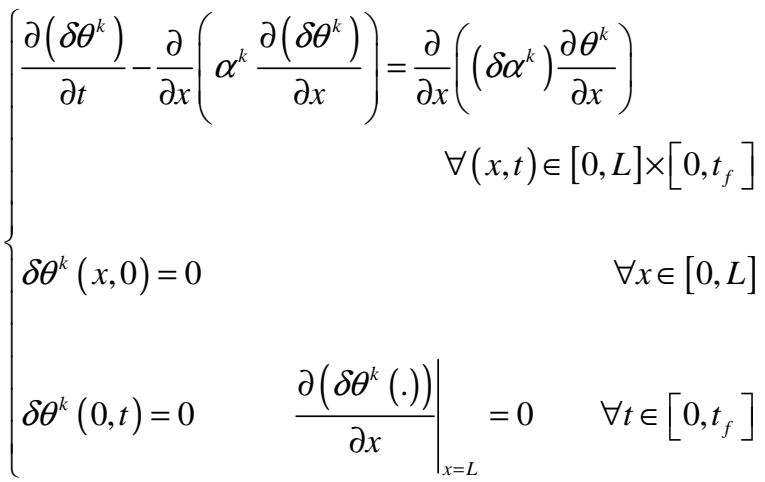

where

$$
\begin{aligned}
\delta \alpha^{k}(x, t) & =\sum_{j=1}^{N t} \sum_{i=1}^{N x}\left(\delta \alpha_{i j}^{k}\right) s_{i}(x) s_{j}(t) \\
& =\sum_{j=1}^{N t} \sum_{i=1}^{N x}\left(d_{i j}^{k}\right) s_{i}(x) s_{j}(t)
\end{aligned}
$$

Descent depth is then defined as follows:

$$
\gamma^{k}=\frac{\int_{0}^{t_{f}} \sum_{i=1}^{N_{s}}\left(\theta^{k}\left(x_{i}, t\right)-\hat{\theta}_{i}(t)\right)\left(\delta \theta^{k}\left(x_{i}, t\right)\right) d t}{\int_{0}^{t_{f}} \sum_{i=1}^{N_{s}}\left(\delta \theta^{k}\left(x_{i}, t\right)\right)^{2} d t}
$$

Previous approach is detailed in [23]. The stop criterion $J_{\text {stop }}$ is the regularizing parameter [2] which acts on the iteration number. If a Gaussian noise $\mathcal{N}(0, \sigma)$ is added on each measurement, then

$$
J_{\text {stop }}=\frac{1}{2} \tilde{N} \sigma^{2} \tau
$$

where $\tilde{N}$ is the number of collected measurements and $\tau$ is the sampling time for measurements. In [24], several restart procedures for the CGM are proposed. It is usual for example to consider $\beta^{k}=0$ when $k=N$. This technique allows refreshment in the calculus of the conjugate direction descent. At each iteration three well posed problems have to be solved: the direct problem (for cost-function evaluation), the adjoint problem (for the gradient evaluation) and the sensitivity problem (for the descent depth estimation). In the following section, several results are presented in a numerical situation.

\section{NUMERICAL RESULTS}

Let us consider the previous direct problem. Numerical simulations obtained according to the thermal diffusivity $\alpha(x, t)$ defined in Table 1 are considered as measurements. Then temperature measurements can be obtained for $N_{s}=11$ sensors located at $x_{i}=\frac{L(i-1)}{10}$ (see Fig. 2) with a sampling time $\tau=1 \mathrm{~s}$. Discretization of unknown parameter $\alpha(x, t)$ is considered according to (2). This discretization depends neither on the sensor numbers nor the sampling time. Without a priori information related to the distribution $\alpha(x, t)$, let us consider $N_{x}=11$ and $N_{t}=9$. It is obvious that this discretization is less accurate than the value $\alpha(x, t)$ defined in Table 1. This model error induces errors in simulated temperatures. In the studied situation, where "measurements" are simulated it is possible to estimate the effect of this model error with a comparison between the solutions of two direct problems (1): the first one with the continuous $\alpha(x, t)$ defined in Table 1 , the second one with 
Attar L., Perez L., Nouailletas R., Moulay E., Autrique L., Thermal diffusivity identification based on an iterative regularization method, IEEE Conference on Decision and Control 2015, Osaka Japan, December 2015.

$\alpha(x, t)=\sum_{j=1}^{9} \sum_{i=1}^{11} \alpha_{i j} s_{i}(x) s_{j}(t) . \quad$ The residual temperatures between these two numerical resolution show that it is not possible to obtain a criterion $J_{\text {stop }}<164$. This threshold is considered for identification without measurement noises.

\section{A. Identification without measurement noises}

Let us consider an initial value for the CGM $\alpha^{0}=\left[\alpha_{i j}^{0}\right]_{N, \times N_{,}}$with $\alpha_{i j}^{0}=0$ for all $i, j$. Cost function evolution versus iteration is shown Fig. 3. Average residual temperature is about $0.19 \mathrm{~K}$ and standard variation is about $1.51 \mathrm{~K}$. Identified thermal diffusivity is presented Fig. 4. which can be compared to Fig. 1. Temperature residuals are small enough to consider that the methodology is efficient. It is important to notice that the algorithm has converged in 53 iterations smaller than the number of unknown parameters (99).

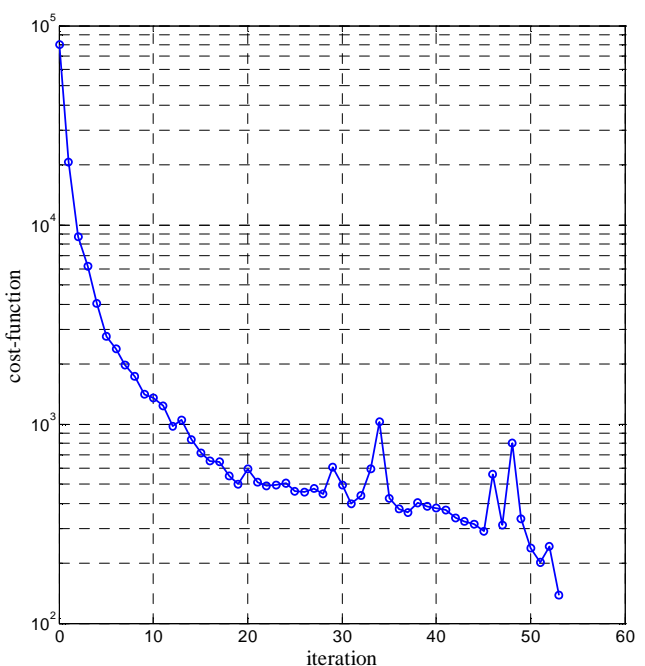

Figure 3. Cost-function evolution without measurement noises

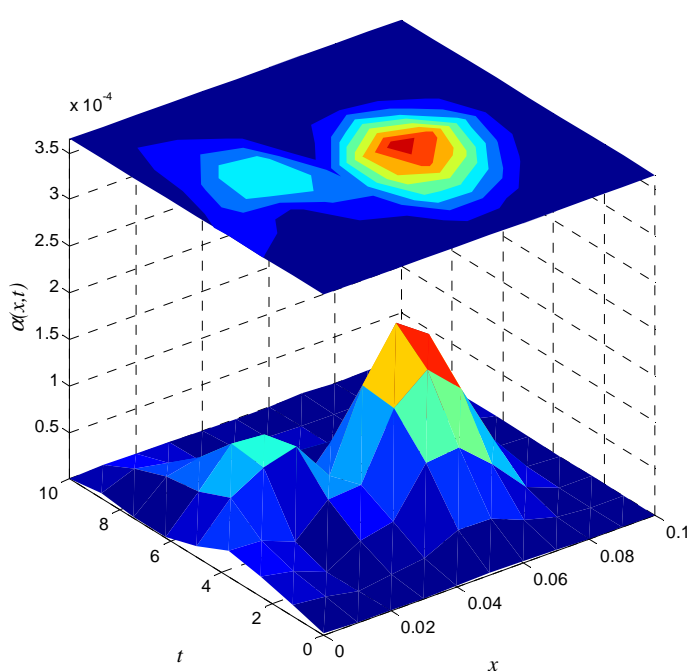

Figure 4. Identified thermal diffusivity at iteration 53 .
This can be easily explained since the real shape of $\alpha(x, t)$ is defined considering less than 99 parameters.

The proposed discretization $\alpha=\left[\alpha_{i j}\right]_{N_{x} \times N_{t}}$ leads to over determination. However the regularizing effect of the CGM is able to overcome this difficulty.

\section{B. Identification with measurement noises}

A Gaussian noise $\mathcal{N}(0,5)$ is considered for measurements, then $J_{\text {stop }}=1513$. In this configuration, cost function evolution versus iteration is shown in Fig. 5.

Average residual temperature is about $0.62 \mathrm{~K}$ and standard variation is about $4.98 \mathrm{~K}$. Identified thermal diffusivity is presented in Fig. 6. With measurement noises, regularizing effect is highlighted: the main structure of the thermal diffusivity is estimated in the first iterations.

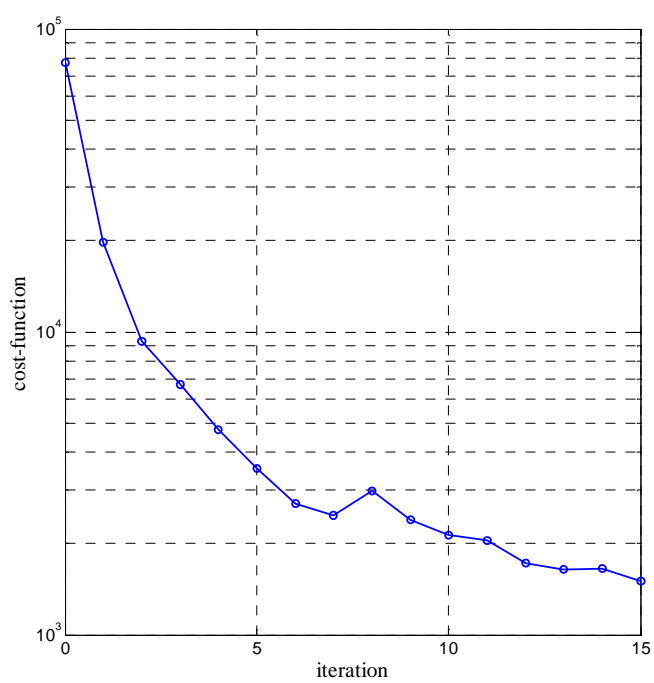

Figure 5. Cost-function evolution with measurement noises

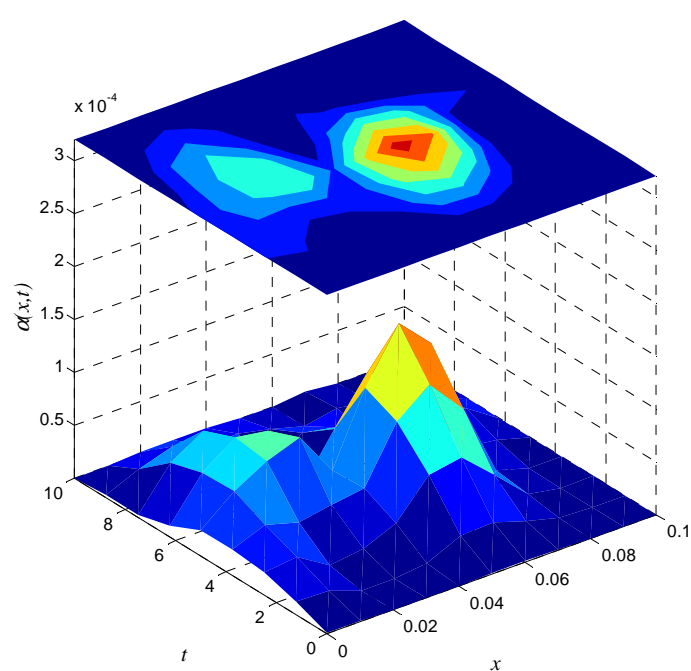

Figure 6. Identified thermal diffusivity at iteration 7. 
Attar L., Perez L., Nouailletas R., Moulay E., Autrique L., Thermal diffusivity identification based on an iterative regularization method, IEEE Conference on Decision and Control 2015, Osaka Japan, December 2015.

\section{CONCLUDING REMARKS AND OUTLOOKS}

Thermal diffusivity identification is a crucial requirement which bring a better understanding of many thermal system behaviors. When the dynamic system state is described by a parabolic partial differential equation system, parametric identification of space and time dependent parameters is not trivial. An approach dedicated to the resolution of ill-posed inverse problem has been proposed. It has been illustrated that both model errors and measurement errors are taken into account in order to sequentially filter perturbations during the identification process. The conjugate gradient algorithm acts as an iterative regularization method where iteration number can be considered as a regularization parameter.

Several outlooks are actually investigated in our institutes. In experimental situations, tracking of moving heating sources (in two-dimensional geometry) using mobile sensors will be based on sequential conjugate gradient method (on sliding time interval). This method seems to provide an attractive alternative to Kalman approach for quasi in-line estimation.

\section{ACKNOWLEDGMENT}

This work was partially supported by the ANR-12-BS03008-03.

\section{REFERENCES}

[1] H.S. Carslaw, and J.C. Jaeger, Conduction of heat in solids, $4^{\text {th }}$ edition, Oxford science publications, 1946.

[2] O.M. Alifanov, Inverse heat transfer problems, Ed. Berlin: SpringerVerlag, 1994.

[3] F. Scarpa, and G. Milano, "Kalman smoothing technique applied to the inverse heat conduction problem," International Journal of Numerical Heat Transfer - Part B, vol. 28, 1995, pp.79-96.

[4] G. Mercere, H. Pálsson, and T. Poinot, "Continuous-time linear parameter-varying identification of a cross flow heat exchanger: a local approach," IEEE Transactions on Control Systems Technology, vol. 19, 2011, pp. 64-76.

[5] W.W. Hager, and H. Zhang, "A survey of nonlinear conjugate gradient method," Pacific journal of Optimization, vol. 2, n¹, 2006, pp. $35-58$.

[6] A. Vergnaud, G. Beaugrand, O. Gaye, L. Perez, P. Lucidarme, and L. Autrique, "Quasi on-line identification of temperature-dependent thermal conductivity," in Proc. European Control Conference 2014, Strasbourg, France, June 2014.
[7] R.M. Mahamood, E.T. Akinlabi, M. Shukla and S. Pityana, "Functionally Graded Material: An Overview," in Proc. of the World Congress on Engineering WCE 2012, London, U.K., July 4 - 6, 2012.

[8] H. Ji, D.P. Sellan, M.T. Pettes, X. Kong, J. Ji, L. Shi, and R.S. Ruoff, "Enhanced thermal conductivity of phase change materials with ultrathin-graphite foams for thermal energy storage," Energy \& environmental sciences, vol. 7, 2014, pp. 1185-1192.

[9] S.I. Braginskii, "Transport processes in a plasma", Rev. Plasma Phys., vol. 1, 1965.

[10] F.L. Hinton, and R.D. Hazeltine, "Theory of plasma transport in toroidal confinement systems", Reviews of Modern Physics, vol. 48, no. 2, Part I, April 1976, pp. 239-308.

[11] F. Bourquin, and A. Nassiopoulos, "Inverse reconstruction of initial and boundary conditions of a heat transfer problem with accurate final state", International Journal of Heat and Mass Transfer, vol. 54, pp. 3749-3760, 2011.

[12] S. Beddiaf, L. Perez, L. Autrique, and J.C. Jolly, "Simultaneous determination of time-varying strength and location of a heating source in a three-dimensional domain", Inverse Problems in Science and Engineering, vol. 22, no. 1, pp. 166-183, 2014.

[13] M.J. Kazemzadeh-Parsi, and F. Daneshmand, "Solution of geometric inverse heat conduction problems by smoothed fixed grid finite element method", Finite Elements in Analysis and Design, vol. 45, pp. 166-183, 2009.

[14] D.W. Pepper, and J.C. Heinrich, The finite element method - basic concepts and applications, Ed. New York: Taylor \& Francis, 2006.

[15] W.B.J. Zimmerman, Multiphysics Modeling With Finite Element Methods, World Scientific Publishing, 2006.

[16] L. Edsberg, Introduction to Computation and Modeling for Differential Equations, Wiley-Interscience, 2008.

[17] A.J. Baker, Finite Elements: Computational Engineering Sciences, Wiley, 2012.

[18] R.W. Pryor, Multiphysics modeling using Comsol® v.4 - a first principles approach, Mercury Learning \& Information, 2012.

[19] V. Isakov, Inverse problems for Partial Differential Equations, Ed. New York: Springer-Verlag, 1998.

[20] V.A. Morozov, Methods for solving incorrectly posed problems, Ed. New York: Springer-Verlag, 1994.

[21] M. Prud'homme, and T. Hung Nguyen, "On the iterative regularization of inverse heat conduction problems by conjugate gradient method", International Communications in Heat and Mass Transfer, vol. 25, no. 7, pp. 999-1008, Oct. 1998.

[22] A. Tarantola, Inverse problem theory and Methods for Model Parameter Estimation, ed. by SIAM Society for Industrial and Applied Mathematics, pp. 342, 2005.

[23] L. Perez, L. Autrique, and M. Gillet, "Implementation of a conjugate gradient algorithm for thermal diffusivity identification in a moving boundaries system", Journal of physics: Conference Series, vol. 135, 2008.

[24] M.J.D. Powell, "Restart procedures for the conjugate gradient method", Mathematical Programming (North-Holland Publishing Company), vol. 12, pp. 241-254, 1977. 